\title{
Avaliação do grau de implantação do Programa Academia da Saúde no município de Bezerros, Pernambuco
}

\author{
Implementation of Health Gym Program in Bezerros city - Brazil
}

\section{AUTORES \\ Rafaela Niels da Silva ${ }^{1,2}$ (D) \\ Janaína Rodrigues de Oliveira ${ }^{3}$ (i) \\ Renata Cecília Barbosa Carneiro ${ }^{4}$ \\ Shirlley Jackllanny Martins de Farias ${ }^{5}$ (i) \\ Flávio Renato Barros da Guarda ${ }^{5}$ (D) \\ 1 Centro Universitário Tabosa de Almeida, Caruaru, Pernambuco, Brasil. \\ 2 Universidade Federal de Pernambuco, Programa de Pós-graduacão em Inovação Terapêutica, Recife, \\ Pernambuco, Brasil. \\ 3 Instituto de Desenvolvimento Educacional, Recife, Pernambuco, Brasil. \\ 4 Faculdade de Comunicação Tecnologia e Turismo Olinda, Pernambuco, Brasil. \\ 5 Universidade Federal de Pernambuco, Centro Acadêmico de Vitória, Vitória de Santo Antão, Pernambuco, Brasil.}

\section{CONTATO}

\section{Rafaela Niels da Silva}

rafaelaniels@gmail.com

Rua Almir Azevedo, n 175, bloco A, apartamento 602, Várzea, Recife, Pernambuco, Brasil.

CEP: 50740-210.

DOI

10.12820/rbafs. $25 \mathrm{e} 0170$

\section{(cc) BY}

Este trabalho está licenciado com uma Licença Creative Commons - Atribuição 4.0 Internacional.

\begin{abstract}
RESUMO
O objetivo deste artigo foi avaliar o grau de implantação do Programa Academia da Saúde (PAS) na cidade de Bezerros, Pernambuco. Estudo descritivo de corte transversal, o qual se caracteriza como uma avaliação normativa da implantação do PAS em suas dimensões de estrutura e processo de trabalho. O estudo foi dividido em quatro etapas: i) validação de um modelo lógico para o programa; ii) elaboração das matrizes de indicadores e julgamento; iii) validação das matrizes por especialistas, e; iv) verificação do grau de implantação através de entrevistas. Utilizou-se um sistema de escores para descrever o grau de implantação como: 1) Incipiente: 0 > 33,3\%; 2) Intermediário: > 33,3 < 66,6\%; 3) Avançado: $>66,6 \%$. O grau de implantação do PAS em Bezerros foi considerado intermediário $(55,0 \%)$, sendo que a dimensão estrutura obteve um escore maior $(64,7 \%)$ que a de processo de trabalho $(48,6 \%)$. Critérios como insumos para a realização das atividades no território e para o aumento do nível de atividade física da população obtiveram pontuação máxima na avaliação, porém, outros elementos de estrutura como a existência de protocolos de ação e monitoramento das ações, assim como de processo de trabalho relativos à mobilização da comunidade e desenvolvimento de ações nas linhas de cuidado obtiveram baixo grau de implantação. O grau de implantação verificado denota a necessidade de qualificação profissional e reorientação das intervenções visando o aperfeiçoamento das atividades de promoção da saúde, e a ampliação das possibilidades de atuação sobre os demais objetivos específicos do programa.
\end{abstract}

Palavras-chave: Sistema Único de Saúde; Avaliação em saúde; Promoção da saúde; Política de saúde. ABSTRACT

The aim of this paper was to analyze the implementation of Health Gym Program in Bezerros city, on Northeastern of Brazil. A cross-sectional study, as a normative evaluation of the implementation of this intervention considering the dimensions of structure and work process. The study was performed through four steps: 1) Validation of a Logic Model to the program;2) development of judgement and indicators matrixes; 3) validation of matrixes by experts, and; 4) interview with stakeholders. The degree of implementation was classified according to a system of scores in: 1) incipient: < 33.3\%; 2) Intermediate: $33.3>66.6 \%$; Advanced: > 66.6\%. The implementation of Health Gym Program in Bezerros is intermediate (55.0\%), but the structure dimension has had a highest score (64.7\%) than work process (48.6\%). Although criteria such as resources to perform activities on the neighborhood and materials to increase the level of physical activity in the population obtained higher score, other elements of structure such as action and monitoring protocols and work process elements related to community mobilization, development of actions on the line of care has obtained low implementation rate. The degree of implementation observed shows the need to professional qualification and restructure of the interventions in order to qualify the health promotion actions and improve the possibilities aim the hole specific objectives of the program.

Keywords: Unified Health System; Health evaluation; Health promotion; Health policy.

\section{Introdução}

Doenças crônicas não transmissíveis (DCNT) representam a principal causa de morbimortalidade no mundo, sendo responsáveis por $63 \%$ dos óbitos mundiais ocorridos em $2008^{1}$. No Brasil, essas doenças apesar da alta prevalência, são de caráter evitável, o que levou o Ministério da Saúde a adotar uma série de ações estratégicas visando diminuir a exposição de indivíduos a comportamentos de risco e promover a adoção de estilos de vida mais ativos e saudáveis, além de atuar na prevenção e controle dessas doenças ${ }^{2,3}$.

Destaca-se neste contexto a criação do Programa Academia da Saúde (PAS) em 2011, o qual visa a construção e custeio de espaços públicos com infraestrutura 
e pessoal qualificado para a promoção da saúde e produção do cuidado através da articulação com serviços de Atenção Primária à Saúde (APS) ${ }^{4}$.

As ações do PAS compõem o escopo das políticas nacionais de Atenção Básica e de Promoção da Saúde, os planos nacionais de enfrentamento de DCNT e intervenções voltadas a atuar sobre os condicionantes modificáveis para essas doenças, caracterizando o programa como uma ação estratégica de promoção no contexto da política pública de saúde no Brasil ${ }^{5}$.

Dada a importância estratégica e o investimento público destinado ao PAS, a avaliação dessa intervenção pode fornecer elementos para o monitoramento, avaliação e (re)orientação das ações, visando a qualidade dos serviços prestados à população ${ }^{6}$.

Entre os vários métodos e desenhos de estudos de avaliação, destaca-se o proposto por Donabedian ${ }^{7}$ o qual é formado pela tríade: estrutura, processo e resultado. A estrutura inclui todos os elementos relativamente estáveis que se destinam à oferta do serviço, tais como os equipamentos, recursos humanos aspectos organizacionais. $\mathrm{O}$ processo está diretamente ligado à execução do conjunto das atividades desenvolvidas no contexto da intervenção ${ }^{8}$, ao passo que os resultados relacionam-se aos produtos das ações e as modificações no estado de saúde de indivíduos e da população ${ }^{7}$. julgamento de valor sobre uma intervenção baseado na comparação desses três componentes com critérios e normas denomina-se avaliação normativa ${ }^{6}$.

A avaliação normativa proporciona um melhor entendimento das variações observadas no que se refere ao nível de implantação ou a integridade de uma dada intervenção $0^{10}$, permitindo verificar sua validade de conteúdo e a intensidade com a qual as atividades são executadas em consonância com as normas existentes $^{6}$. Esse tipo de estudo é adequado para intervenções complexas $^{6}$, como é o caso do PAS, o qual visa intervir sobre uma série de condicionantes da saúde, articulando diferentes atores e setores da sociedade 9 .

A avaliação do grau de implantação do PAS pode, portanto, contribuir para a melhoria da qualidade das ações ofertadas, identificando possíveis discrepâncias entre as ações preconizadas pelo Ministério da Saúde e a forma como o programa é desenvolvido no município, configurando-se como uma importante ferramenta para a tomada de decisão em gestão ${ }^{6}$.

O município de Bezerros, situado na mesorregião do Agreste Pernambucano, apresenta em seu perfil epidemiológico altas prevalências de $\mathrm{DCNT}^{10}$. Nesse sen- tido, implantou desde 2013 um polo do PAS em uma comunidade da zona rural, visando ampliar o escopo das ações de promoção da saúde na atenção primária ${ }^{10}$.

A implantação do PAS em Bezerros, assim como nos demais municípios brasileiros referencia-se nas diretrizes oficiais do programa, estabelecidas pelo $\mathrm{Mi}$ nistério da Saúde ${ }^{11,12}$. Entretanto, de acordo com Silva et al. ${ }^{9}$, as portarias que regulamentam a organização e funcionamento do PAS parecem não ser suficientemente claras para subsidiar o seu processo de implantação nos municípios, o que pode comprometer tanto a execução das ações, quanto o alcance dos objetivos previstos para essa intervenção.

Diante desse contexto, considerando que o PAS é um programa estratégico para a promoção da saúde e produção do cuidado, com alto investimento financeiro para o gestor federal do Sistema Único de Saúde (SUS) e com necessidade de contrapartida de diversos insumos por parte dos municípios ${ }^{5}$, a avaliação do seu grau de implantação pode fornecer elementos para a melhoria da sua estrutura e otimização dos processos de trabalho, além de identificar como a relação entre esses dois componentes pode influenciar nos resultados dessa intervenção. Dessa forma, o objetivo deste artigo foi avaliar o grau de implantação do PAS na cidade de Bezerros, Pernambuco.

\section{Método}

Realizou-se um estudo descritivo de corte transversal com abordagem quantitativa, o qual se caracteriza como uma avaliação normativa da implantação do Programa Academia da Saúde no município de Bezerros, buscando verificar aspectos inerentes à estrutura e processo de trabalho.

O município de Bezerros está situado no Agreste Pernambucano, possui uma população estimada em 60.469 habitantes e está localizado a $102 \mathrm{~km}$ da capital do estado ${ }^{13}$. Seu Produto Interno Bruto per capita em 2017 era de $\mathrm{R} \$ 11.752,51$ (53 do estado) e o Índice de Desenvolvimento Humano, de acordo com o último censo é de 0,606. A rede de serviços de saúde do município é formada por 22 Unidades de Saúde da Família, um Centro de Reabilitação, uma Policlínica, uma Farmácia 24 horas, um Centro de Apoio Psicossocial, um Núcleo de Apoio à Saúde da Família, um polo do PAS, uma Clínica da Mulher, um Centro de Especialidade Odontológica, uma Unidade Mista e uma Unidade Móvel SAMU. A população assistida na Estratégia de Saúde da Família é de 58,6\% ${ }^{14}$. 
O PAS foi implantado com apenas um polo em Bezerros, no ano de 2013. Nesse polo atuam duas profissionais de educação física, que realizam seções diárias de atividade física orientada (aulas de ginástica) nos turnos da manhã (06:00 às 08:00h) e da tarde (17:00 às 19:00h), além de rodas de conversa quinzenais sobre temas relacionados à saúde e qualidade de vida. Essas atividades eventualmente contam com a presença da nutricionista do Núcleo de Apoio à Saúde da Família (NASF), a qual desenvolve ações pontuais de orientação nutricional.

Este estudo foi dividido em cinco etapas: I - Validação de um modelo lógico proposto para o PAS por parte dos gestores e executores do programa no município de Bezerros; II - Elaboração das matrizes de indicadores e julgamento com base nas atividades previstas para o programa; III - Validação das matrizes por especialistas, através da realização de um painel de consenso; IV - Verificação do grau de implantação do PAS.

\section{- Etapa I - validação de um modelo lógico para o} programa: o modelo lógico é uma representação gráfica da relação entre os elementos necessários à operacionalização da política, visando o alcance dos resultados esperados ${ }^{6}$. Para este estudo utilizou-se o modelo lógico proposto por Silva et al. ${ }^{9}$, o qual foi desenvolvido através de um estudo de aviabilidade do PAS no município do Recife (Pernambuco), a partir de portarias, diretrizes e demais documentos oficiais referentes à Academia da Saúde.

Esse modelo foi avaliado pelos pesquisadores e submetido à apreciação do profissional de educação física que atua no programa, da coordenadora da Gerência de Atenção à Saúde, coordenadora do NASF e coordenadora do PAS. Essas foram submetidas a uma seção de treinamento (na sede da secretaria de saúde do município), na qual os pesquisadores informaram a respeito de como realizar a validação do modelo por meio da análise dos componentes (existência e pertinência), análise lógica (coerência da relação entre os componentes) e o teste de consistência do modelo lógico, conforme as estratégias de validação propostas por Hartz \& Silva ${ }^{6}$.

O processo de validação foi realizado de forma coletiva, através de uma seção na qual os elementos do modelo foram apresentados, descritos e discutidos pelos pesquisadores e informantes-chave, quanto à sua presença, aplicação no contexto do PAS no município e coerência quanto às suas inter-relações e o potencial alcance dos objetivos do programa.

- Etapa II - Elaboração das matrizes de indicadores e julgamento com base nas atividades previstas para o programa: a construção das matrizes de estrutura e processo de trabalho foi realizada com base no modelo lógico do programa, através da realização de três reuniões da equipe de pesquisadores. Os componentes e/ou subcomponentes descritos no modelo lógico foram utilizados como referência para identificar a estrutura necessária (recursos humanos e materiais) e o processo adequado (ações a serem realizadas), para alcançar resultados de curto e médio prazos que caracterizariam a contribuição do programa para a promoção da saúde e produção do cuidado no território ${ }^{6}$.

As matrizes utilizam um sistema de escores, o qual atribui uma pontuação específica para cada indicador proposto e incluem indicadores relativos aos insumos, atividades, produtos e resultados que podem ser esperados do programa em curto e médio prazo, utilizando como fontes de dados os registros sistemáticos do programa, sistemas de informações e informantes-chaves (profissionais e gestores do programa).

As matrizes são compostas pelos critérios de avaliação, indicadores, padrões de verificação, pontuação estabelecida para cada indicador e pelo valor observado no programa, através da aplicação de questionário junto aos informantes-chave. As matrizes de estrutura e processo para a avaliação do grau de implantação do PAS em Bezerros podem ser consultadas no material de apoio (apêndice) a este artigo.

- Etapa III - Validação das matrizes de indicadores e julgamento: a estratégia adotada para a validação das matrizes foi a realização de um painel de consenso ${ }^{10}$. Para tanto, foram convidadas, exclusivamente para esta etapa, quatro profissionais de saúde, todas com pós-graduação em saúde pública, sendo duas doutoras e duas mestras. Essas foram convidadas a participar do processo de validação das matrizes por e-mail, devido às suas experiências nos campos da avaliação em saúde e da atividade física, por sua produção científica relevante (livros e artigos publicados) nos últimos cinco anos e por terem participado do processo de construção e validação do modelo lógico do PAS para outro município pernambucano.

A essas especialistas foram enviados: as matrizes de estrutura e processo de trabalho, as portarias 
normativas do programa e o modelo lógico validado pelos profissionais e gestores do município de Bezerros que participaram das etapas I e IV deste estudo (validação do modelo lógico). Não houve necessidade de uma segunda apreciação por parte das especialistas, pois as correções requeridas foram muito semelhantes e relacionavam-se aos mesmos temas, tais como a inclusão de critérios relativos à presença de instrumentos de monitoramento e avaliação do programa.

- Etapa IV - Verificação do grau de implantação do PAS: com base no modelo lógico e nas matrizes de indicadores e julgamento foi elaborado o roteiro de entrevista semiestruturado, com perguntas relativas à estrutura e ao processo de trabalho do PAS. Após a realização de um treinamento para a aplicação do instrumento, esse foi respondido entre novembro de 2016 e fevereiro de 2017, na sede da secretaria de saúde, pela gestora de Atenção à Saúde, pela coordenadora do NASF, pela coordenadora do PAS, e no polo do programa pela profissional de educação física que atua no PAS de Bezerros.

O processamento e a análise das respostas à entrevista foram realizados manualmente. Nos casos de discrepância entre as respostas apresentadas pelos diferentes informantes-chave, foram consideradas as observações que pudessem ser comprovadas através de fontes de verificação como: atas de reuniões, protocolos, documentos impressos e fotografias, bem como a apresentação de materiais e/ou insumos. Após a checagem e confirmação das fontes de verificação, os resultados foram transcritos para as matrizes de indicadores e julgamento para a realização do cálculo do grau de implantação.

Os critérios para julgamento do grau de implantação basearam-se nos instrumentos normativos do programa, tais como: portarias de redefinição e custeio $^{11,12}$, as políticas nacionais de Atenção Básica ${ }^{15}$ e de promoção da saúde ${ }^{16}$, além do modelo lógico do programa proposto por Silva et al. ${ }^{9}$.

A partir das informações coletadas por meio das entrevistas, o grau de implantação do Programa Academia da Saúde foi obtido através do preenchimento das matrizes de indicadores e julgamento.

No processo de análise das matrizes e cálculo do grau de implantação foi utilizado um sistema de escores que concedeu uma pontuação individual para cada indicador, multiplicou esse valor pelo número indicadores em cada critério e utilizou uma regra de três para calcular o percentual do grau de implantação para cada critério e para os elementos de estrutura e de processo de trabalho.

Considerando que a matriz de estrutura foi composta por 44 indicadores, e que a de processo possuía 41, a implantação total do PAS seria representada por escores iguais a $100 \%$ tanto para a estrutura, quanto para o processo. Neste caso, os valores referentes a cada indicador de estrutura foram de 2,27 e a pontuação referente a cada indicador de processo foi 2,44 . A equação que foi utilizada para o cálculo do valor a ser atribuído individualmente a cada indicador (de estrutura ou de processo) é descrita a seguir:

VII $=\frac{\text { NrInd }}{100}$

Onde VII é o valor individual a ser atribuído a um indicador de estrutura ou processo; NrInd corresponde ao número total de indicadores na respectiva matriz, e; 100 corresponde máxima pontuação que pode ser obtida na avaliação do grau de implantação. O grau de implantação para cada critério dos elementos de estrutura e processo foi obtido através de uma regra de três simples, conforme a equação a seguir:

$\mathbf{G I C}_{\mathbf{i}}=\frac{\mathrm{VII} * \mathrm{n} * 100}{\mathrm{PMPC}}$

Onde $\mathrm{GIC}_{i}$ representa o grau de implantação de um critério $i$, VII é o valor individual do indicador (conforme descrito na equação 1 ), $n$ representa o número de indicadores em cada critério, 100 é o valor da constante para o cálculo do percentual de implantação, e PMPC é a pontuação máxima prevista para um determinado critério constante na matriz. Para o cálculo final do grau de implantação, os itens referentes à estrutura receberam peso quatro e os de processo de trabalho, peso seis, conforme a equação a seguir:

GIMP $=\frac{\left[\left(\sum P O E \times 100\right) \div 44\right] \times 4+\left[\left(\sum P O P \times 100\right) \div 41 \times 6\right]}{10}$

Onde GIMP representa o grau de implantação do programa, $\mathrm{POE}$ e $\mathrm{POP}$ representam, respectivamente, a pontuação obtida nos itens de estrutura e processo de trabalho, 44 é o número total de indicadores de estrutura, 4 é o peso atribuído ao componente de estrutura, 41 é o número total de indicadores de processo, 6 é o peso atribuído ao componente 
processo de trabalho e 100 é o valor da constante para o cálculo do percentual de implantação.

A atribuição dos pesos teve caráter arbitrário e se deu diante da importância que cada um possui para que se promova a oferta das ações do Programa, considerando que ter a estrutura para o adequado desenvolvimento das atividades é muito importante, mas não implica necessariamente na oferta de boa qualidade, caso o processo de trabalho não seja um fator determinante que agregue uma consciência de maior importância para os profissionais na execução de suas ações e atividades desenvolvidas no Programa ${ }^{6,18}$.

Por fim, o grau de implantação do programa foi definido de acordo com pontos de corte distribuídos em tercis $^{17}$ : (1) Incipiente: $0>33,3 \%$; (2) Intermediário: > 33,3 < 66,6\%; (3) Avançado: > 66,6\%.

$\mathrm{O}$ projeto de pesquisa referente a este estudo foi aprovado pelo Comitê de Ética em Pesquisa do Centro de Ciências da Saúde da Universidade Federal de Pernambuco através do parecer no 1.744.232, de 26 de setembro de 2016. Todos os participantes assinaram Termo de Consentimento Livre e Esclarecido.

\section{Resultados}

O grau de implantação do Programa Academia da Saúde em Bezerros foi considerado intermediário (55,0\%), sendo que a dimensão estrutura obteve um escore maior $(64,7 \%)$ que a de processo de trabalho $(48,6 \%)$.

Para a descrição dos resultados da avalição do grau de implantação, a pontuação tanto dos indicadores de estrutura, quanto dos de processo de trabalho foi agrupada dentro dos critérios descritos na matriz de julgamento e apresentados sob a forma de quadros. Esses critérios correspondem aos componentes (ou subcomponentes) propostos no modelo lógico do programa.

O Quadro 1 descreve a quantidade de indicadores que compõem os critérios de estrutura e processo de trabalho do PAS em Bezerros.

A dimensão da estrutura foi avaliada de acordo com o espaço físico, recursos humanos, equipamentos e material, existência de protocolos e diretrizes de funcionamento, sendo sua matriz composta por 44 indicadores.

Os critérios, pontuação máxima para cada critério, a pontuação obtida a partir da aplicação das entrevistas, o percentual de cumprimento das atividades dentro de cada critério e grau de implantação referentes à dimensão da estrutura do PAS em Bezerros são descritos na Tabela 1.
Quadro 1 - Quantidade de indicadores que compõem os critérios de estrutura e processo de trabalho do PAS - Bezerros, Pernambuco, 2017.

\begin{tabular}{|lcc|}
\hline Critério & $\begin{array}{c}\text { Indicadores } \\
\text { de estrutura }\end{array}$ & $\begin{array}{c}\text { Indicadores } \\
\text { de processo }\end{array}$ \\
\hline Ações de gestão & 2 & 6 \\
$\begin{array}{l}\text { Ações de promoção da saúde no contexto da } \\
\text { atenção básica }\end{array}$ & 2 & 3 \\
Ações intersetoriais & 2 & 5 \\
$\begin{array}{l}\text { Produção do cuidado integral a partir das } \\
\text { linhas de cuidado }\end{array}$ & 2 & 3 \\
Ações de educação em saúde & 2 & 2 \\
Mobilização da comunidade & 2 & 3 \\
Integração multiprofissional & 3 & 6 \\
Aumento dos níveis de atividade física & 4 & 5 \\
Promoção de hábitos alimentares saudáveis & 2 & 3 \\
$\begin{array}{l}\text { Ações de inclusão social e enfrentamento de } \\
\text { violências }\end{array}$ & 1 & 1 \\
Recursos humanos & 8 & 1 \\
$\begin{array}{l}\text { Insumos necessários e adequados ao } \\
\text { desenvolvimento das atividades do programa }\end{array}$ & 9 & 0 \\
Registro de procedimentos & 5 & 3 \\
\hline
\end{tabular}

Entre os indicadores de estrutura que obtiveram escore zero destacam-se: inexistência do grupo de apoio à gestão e a ausência de protocolos para o mapeamento do território, acolhimento e humanização da atenção. Observou-se, ainda, que não existem fóruns que agreguem outros programas da rede de saúde e demais atores no processo do cuidado.

Ainda foi possível verificar a inexistência de protocolos de definição de atividades e responsabilidades para o desenvolvimento de ações de integração entre as diferentes categorias profissionais que atuam no programa; a inexistência de instrumentos para avaliar os níveis de atividade física na população; ausência de planejamento para as ações de inclusão social e enfrentamento de violências; o baixo percentual de profissionais do NASF atuando no programa e; a falta de materiais necessários para o desenvolvimento de ações de mobilização da comunidade como (cartazes, panfletos, álbuns seriados, equipamentos multimídia dentre outros).

Entre os aspectos positivos relacionados à estrutura, destacam-se: a existência de um plano de ação do programa com foco na promoção da saúde através da atividade física, o registro dos atores envolvidos nessas ações, tais como: enfermeiros, técnicos de enfermagem profissionais de educação física e a pactuação de atividades com o setor da assistência social do município.

A dimensão do processo de trabalho foi avaliada de acordo com as ações e atividades relativas à promoção 
da saúde e produção do cuidado no PAS, sendo composta por 41 indicadores.

Os critérios, pontuação para cada critério, pontuação obtida através da entrevista, percentual de cumprimento das atividades e grau de implantação referentes à dimensão do processo de trabalho são descritas na Tabela 2.

Entre os componentes com maior grau de implantação destacam-se os indicadores: percentual de profissionais que participaram de atividades de formação continuada e/ou em serviço no último ano, participação de ao menos um profissional da Rede de Atenção à Saúde (RAS) em atividades de formação continuada e em serviço sobre promoção da saúde ou sobre as ações do programa no último ano.

Observou-se também o quantitativo de profissionais que desenvolveu atividades em parceria com a comunidade e com outros pontos da RAS no último mês. Cabe ressaltar, entretanto, que essa atividade se limitou à realização das atividades cotidianas dos profissionais de educação física, eventualmente apoiadas pela enfermeira ou técnica de enfermagem da Unidade de Saúde localizada no território onde o polo se encontra.

Outro indicador positivo foi a realização de uma atividade de inclusão social e enfrentamento de violência nos últimos seis meses. Segundo os profissionais de educação física que atuam no PAS, as atividades físicas

Tabela 1 - Critérios, pontuação máxima, pontuação obtida, percentual de cumprimento das atividades e grau de implantação da dimensão da estrutura do PAS - Bezerros Pernambuco, 2017.

\begin{tabular}{|c|c|c|c|c|}
\hline Critérios & $\begin{array}{l}\text { Pontuação } \\
\text { Máxima }\end{array}$ & $\begin{array}{c}\text { Pontuação } \\
\text { Obtida }\end{array}$ & $\begin{array}{l}\text { Cumprimento } \\
(\%)\end{array}$ & $\begin{array}{c}\text { Grau de } \\
\text { Implantação }\end{array}$ \\
\hline Ações de gestão & 4,54 & 4,54 & 100,0 & Avançado \\
\hline Ações de promoção da saúde no contexto da atenção básica & 4,54 & 2,27 & 50,0 & Intermediário \\
\hline Ações intersetoriais & 4,54 & 2,27 & 50,0 & Intermediário \\
\hline Produção do cuidado integral a partir das linhas de cuidado & 4,54 & 2,27 & 50,0 & Intermediário \\
\hline Ações de educação em saúde & 4,54 & 2,27 & 50,0 & Intermediário \\
\hline Mobilização da comunidade & 4,54 & 0 & 0,0 & Incipiente \\
\hline Integração multiprofissional & 6,81 & 1,15 & 16,9 & Incipiente \\
\hline Aumento dos níveis de atividade física & 9,08 & 6,81 & 75,0 & Avançado \\
\hline Promoção de hábitos alimentares saudáveis & 4,54 & 4,54 & 100,0 & Avançado \\
\hline Ações de inclusão social e enfrentamento de violências & 2,27 & 0 & 0,0 & Incipiente \\
\hline Recursos humanos & 18,16 & 15,84 & 87,2 & Avançado \\
\hline $\begin{array}{l}\text { Insumos necessários e adequados ao desenvolvimento das atividades do } \\
\text { programa }\end{array}$ & 20,43 & 15,89 & 77,8 & Avançado \\
\hline Registro de procedimentos & 11,35 & 6,81 & 40,0 & Avançado \\
\hline
\end{tabular}

Tabela 2 - Critérios, pontuação máxima, pontuação obtida, percentual de cumprimento das atividades e grau de implantação da dimensão processo de trabalho do PAS - Bezerros, Pernambuco, 2017.

\begin{tabular}{|c|c|c|c|c|}
\hline Critérios & $\begin{array}{c}\text { Pontuação } \\
\text { Máxima }\end{array}$ & $\begin{array}{c}\text { Pontuação } \\
\text { Obtida }\end{array}$ & $\begin{array}{c}\text { Cumprimento } \\
(\%)\end{array}$ & $\begin{array}{c}\text { Grau de } \\
\text { Implantação }\end{array}$ \\
\hline Ações de gestão & 14,64 & 12,2 & 83,3 & Avançado \\
\hline Ações de promoção da saúde no contexto da atenção básica & 7,32 & 7,32 & 100,0 & Avançado \\
\hline Ações intersetoriais & 12,2 & 6,71 & 55,0 & Intermediário \\
\hline Produção do cuidado integral a partir das linhas de cuidado & 7,32 & 2,44 & 33,3 & Incipiente \\
\hline Ações de educação em saúde & 4,88 & 2,44 & 50,0 & Intermediário \\
\hline Mobilização da comunidade & 7,32 & 0 & 0,0 & Incipiente \\
\hline Integração multiprofissional & 14,64 & 2,88 & 19,7 & Incipiente \\
\hline Aumento dos níveis de atividade física & 12,2 & 6,5 & 53,3 & Intermediário \\
\hline Promoção de hábitos alimentares saudáveis & 7,32 & 2,44 & 33,3 & Incipiente \\
\hline Ações de inclusão social e enfrentamento de violências & 2,44 & 2,44 & 100,0 & Avançado \\
\hline Recursos humanos & 2,44 & 0 & 0,0 & Incipiente \\
\hline Registro de procedimentos & 7,32 & 3,25 & 44,4 & Intermediário \\
\hline
\end{tabular}


desenvolvidas no polo caracterizam-se como inclusivas e de prevenção de violência.

Também foram considerados incipientes os indicadores: realização de no mínimo uma atividade mensal de educação em saúde no território; existência de no mínimo uma atividade de mobilização da comunidade nos últimos seis meses e; participação dos profissionais do programa em pelo menos uma reunião intersetorial no último ano, o que impactou sobre as atividades direcionadas à população envolvendo outros setores e parceiros, bem como realização de oficinas para integração de saberes e práticas profissionais no cotidiano dos serviços (Estratégia Saúde da Família-ESF e NASF) nos últimos seis meses, que também contribuíram para o baixo grau de implantação em seus respectivos critérios/componentes.

No que cabe aos critérios aumento dos níveis de atividade física e promoção de hábitos alimentares saudáveis, observou-se que não foi realizado o levantamento dos níveis de atividade física da população, bem como dos condicionantes e determinantes da prática de atividade física no último ano; a não realização de no mínimo uma atividade de alimentação saudável no território uma vez por semana e; o baixo percentual de profissionais do NASF que desenvolvem atividades no PAS.

\section{Discussão}

A avaliação normativa de intervenções em saúde pública configura-se como uma importante ferramenta para a tomada de decisão e avanço no campo científi$\mathrm{co}^{6}$, pois permite comparar os serviços oferecidos com critérios e normas predeterminadas para o alcance dos resultados esperados, subsidiando a realização de estudos avaliativos mais complexos ${ }^{18}$, inclusive sobre os investimentos financeiros e custo-efetividade desses programas e ações ${ }^{19}$.

O maior grau de implantação da dimensão estrutura em relação ao processo de trabalho pode ter relação com o fato de que a presença de elementos de estrutura não implica necessariamente na execução satisfatória dos processos de trabalho ou em proporção equivalente aos recursos existentes ${ }^{6}$.

No caso particular do PAS, a complexidade do seu objetivo geral (contribuir para a promoção da saúde), assim como o grande número de objetivos específicos, princípios e diretrizes para a sua execução pode dificultar o processo de implantação do programa no âmbito do município. Essa dificuldade já foi apontada por Silva e colaboradores ${ }^{9}$ em um estudo sobre a ava- liabilidade do PAS em Recife, Pernambuco, e se reflete no grande número de indicadores para a avaliação da estrutura $(n=44)$ e do processo de trabalho $(n=41)$, necessários para a verificação do grau de implantação na cidade de Bezerros.

As atividades do PAS em Bezerros são essencialmente desenvolvidas por profissionais de educação física, com algumas intervenções pontuais da nutricionista do NASF. Embora essas duas categorias profissionais estejam previstas no rol de trabalhadores que podem atuar no programa ${ }^{12}$ e reflitam o predomínio desses profissionais em outros estudos ${ }^{4,20}$, convém ressaltar que por tratar-se o PAS de um programa de promoção da saúde no contexto da atenção básica, a ausência de outros profissionais nesse processo pode comprometer a integralidade do cuidado e representa um distanciamento da normativa do programa, das diretrizes da atenção básica e dos princípios da promoção da saúde ${ }^{20}$.

O desenvolvimento de ações pontuais no programa por parte da nutricionista compromete a continuidade das ações e estabelecimento de vínculos entre a população e o serviço. Entretanto, estudos que avaliaram atividades de promoção da saúde na Atenção Básica ${ }^{21}$ e especificamente no $\mathrm{PAS}^{22}$, já apontaram essa prática.

No que se refere à implantação da dimensão da estrutura, verifica-se que alguns dos indicadores descritos como incipientes no PAS em Bezerros também foram descritos em outros estudos sobre programas de atividade física. A ausência de instrumentos de monitoramento das ações já foi apontada por Guarda et al. ${ }^{22}$ ao avaliar o PAS em quatro municípios da Região Metropolitana do Recife. Esses mesmos autores apontam a inexistência de protocolos de ação e de instrumentos para a avaliação no nível de atividade física da população, que também foi verificada como um indicador crítico do programa em Bezerros.

A ausência de instrumentos de planejamento para o desenvolvimento das ações intersetoriais verificada no PAS em Bezerros, também foi identificada na cidade do Recife $^{9}$ e em outros municípios pernambucanos ${ }^{22}$. Esses mesmos estudos referem uma pequena quantidade de profissionais dos NASF apoiando o programa, e insuficiência de recursos materiais, assim como verificado em Bezerros.

Por outro lado, a existência de um plano de ação do programa com foco na promoção da saúde através da atividade física, bem como o registro dos atores envolvidos nessas ações denota um maior direcionamento de recursos do PAS para o cumprimento do seu décimo 
objetivo específico, o qual visa aumentar os níveis de atividade física da população ${ }^{11}$. Nesse sentido, a implantação desse critério aponta ainda para o fortalecimento das ações previstas no Plano de Ações Estratégicas para o Enfrentamento das DCNT no Brasil ${ }^{5}$. Por outro lado, a concentração de insumos nesse critério e a implantação incipiente no que se refere à integração multiprofissional e em ações de inclusão social e enfrentamento de violências pode caracterizar a fragmentação do cuidado e pouca efetividade das ações ${ }^{20}$.

A avaliação da dimensão do processo de trabalho possibilita saber em que medida as atividades desenvolvidas no programa são adequadas para atingir os resultados esperados ${ }^{6}$. $\mathrm{Na}$ apreciação dessa dimensão no município de Bezerros, destacam-se positivamente os indicadores referentes à participação dos profissionais de educação física em atividades de formação continuada e/ou em serviço no último ano, bem como a participação de ao menos um profissional da Rede de Atenção à Saúde em atividades de formação continuada e em serviço sobre promoção da saúde ou sobre as ações do programa no último ano. Essa participação em treinamentos específicos sobre o programa ou sobre a promoção da saúde qualifica a intervenção e aumenta a efetividade das ações junto à populaçãa ${ }^{23}$.

Dois outros indicadores avaliados positivamente foram a realização de intervenções de incentivo à prática atividade física em parceria com a comunidade e com outros pontos da Rede de Atenção à Saúde, e a realização de uma atividade de inclusão social e enfrentamento de violência. Embora essas ações denotem um cenário de boa articulação multiprofissional e intersetorial do programa avaliado em relação a outros programas de promoção da atividade física em diferentes localidades ${ }^{9}$, essas ainda se mostram incipientes em relação à totalidade dos objetivos e diretrizes propostos para o PAS, os quais apontam a necessidade de constante articulação intra e intersetorial, tanto no contexto das RAS, quanto na integração das ações com setores como educação, cultura, esporte e lazer ${ }^{3,11}$.

A falta de articulação com outros serviços de saúde na rede municipal no que se refere à produção do cuidado integral a partir das linhas de cuidado em Bezerros também foi identificada em outras cidades brasileiras $^{4,9}$. Esse cenário pode apresentar impacto negativo sobre a integralidade do cuidado, pois a articulação com os demais componentes da RAS caracteriza-se como um elemento fundamental para o estabelecimento de prioridades, adequação das ações ao perfil epidemioló- gico da comunidade e construção de linhas de cuidado efetivas $^{4,22}$.

No que cabe aos indicadores relativos aos critérios de aumento dos níveis de atividade física e promoção de hábitos alimentares saudáveis, esses também se assemelham aos resultados de outros estudos sobre processo de trabalho no $\mathrm{PAS}^{20,22}$. Cabe ressaltar, entretanto, que esses dois critérios são descritos por Sá et al. ${ }^{23}$ como as atividades mais desenvolvidas pelo PAS no Brasil. Nesse sentido, a não identificação dos níveis de atividade física da população, bem como dos condicionantes e determinantes dessa prática apontam para uma dificuldade de se monitorar essas ações e verificar o cumprimento desse objetivo específico do programa.

Esta avaliação não verificou os elementos dos contextos político-normativo, técnico-gerencial e organizacional que podem relacionar-se ao grau de implantação do programa no município investigado, o que se caracteriza como uma limitação deste estudo. Assim sendo, sugere-se o desenvolvimento de estudos de análise de implantação do PAS, cuja abordagem metodológica permite identificar a influência dos elementos do contexto sobre a operacionalização de uma intervenção em saúde.

A avaliação do grau de implantação do Academia da Saúde em Bezerros demonstra que pouco mais da metade da estrutura e processos de trabalho desenvolvidas no município alinham-se às diretrizes preconizadas pelo Ministério da Saúde para essa intervenção.

No que cabe à dimensão de estrutura, destaca-se a ausência de protocolos de ação e instrumentos de monitoramento das atividades, visando orientar o trabalho dos profissionais e demais elementos da rede de atenção do município que devem articular-se com a equipe do programa. Quanto à dimensão de processo de trabalho, o baixo grau de implantação verificado denota uma limitada participação do controle social, pequena abrangência das atividades voltadas à integralidade do cuidado, além da necessidade de reorientação das ações com vista à caracterização do programa como referência para a promoção da saúde, prevenção e atenção às DCNT, conforme descrito nas diretrizes do programa.

Políticas e programas públicos de saúde demandam a utilização de recursos os quais são finitos e que normalmente são investidos em algumas ações em detrimento da sua alocação em outras atividades preventivas ou assistenciais. Nesse sentido, baixos níveis de implantação podem impactar nos resultados propostos para a intervenção e consequentemente na qualidade 
dos serviços prestados à população, além de gerar questionamentos acerca da manutenção do investimento no programa, em face dos seus custos financeiros nas três esferas de governo.

\section{Conflito de interesse}

Os autores declaram não haver conflito de interesse.

\section{Contribuição dos autores}

Silva RN, participou da elaboração do projeto, da revisão da literatura, da redação e revisão do manuscrito. Oliveira JR, participou da revisão da literatura e da revisão do manuscrito. Carneiro $\mathrm{RCB}$, orientou a elaboração do projeto, sugeriu elementos da revisão da literatura e revisou o texto. Farias SJM, sugeriu elementos da revisão da literatura e revisou o texto. Guarda FRB, orientou a elaboração do projeto, sugeriu elementos da revisão da literatura e revisou o texto.

\section{Agradecimentos}

Os autores agradecem a Secretaria Municipal de Saúde de Bezerros pelo apoio e colaboração com a pesquisa.

\section{Referências}

1. Wang H, NaghavI M, Allen C, Barber RM, Abhutta Z, Carter A, et al. Global, regional, and national life expectancy, allcause mortality, and cause-specific mortality for 249 causes of death, 1980-2015: a systematic analysis for the global burden of disease study 2015. Lancet. 2016;388(10053):1459-44.

2. Malta DC, Bernal RTI, Lima MG, Araújo SSC, Silva MMA, Freitas MIF, et al. Doenças crônicas não transmissíveis e a utilização de serviços de saúde: análise da Pesquisa Nacional de Saúde no Brasil. Rev. Saúde Pública. 2017;51(Suppl 1):S4.

3. Malta DC, Silva MMA, Albuquerque GM, Lima CM, Cavalcante T,Jaime PC, et al.A implementação das prioridades da Política Nacional de Promoção da Saúde, um balanço, 2006 a 2014. Cien Saude Colet. 2014;19(11):4301-12.

4. Sá GBAR, Dornelles GC, Cruz KG, Amorim RCA, Andrade SSCA, Oliveira TP, et al. O Programa Academia da Saúde como estratégia de promoção da saúde e modos de vida saudáveis: cenário nacional de implementação. Cien Saude Colet. 2016;21(6):1849-60.

5. Malta DC, Oliveira TP, Santos MAS, Andrade SSCA, Silva MMA. Progress with the Strategic Action Plan for Tackling Chronic Non-Communicable Diseases in Brazil, 2011-2015. Epidemiol. Serv. Saúde. 2016;25(2):373-90.

6. Hartz ZMA, Silva LMV. Avaliação em Saúde: dos modelos teóricos à prática na avaliação de programas e sistemas de saúde.4ª ed. Rio de Janeiro: Editora Fiocruz, 2014.

7. Donabedian A. Explorations in quality assessment and monitoring. $3^{a}$ ed.Ann Arbor:Health Administration Press, 1988.

8. Donabedian A. Evaluating the quality of medical care. Milbank Q. 2005;83(4):691-729.

9. Silva RN, Guarda FRB, Hallal PC, Martelli PJ L. Avaliabilidade do Programa Academia da Saúde no Município do Recife, Pernambuco, Brasil. Cad Saúde Pública.2017;33(4): e00159415.
10. DATASUS. Morbidade hospitalar do SUS - por local de residência -Pernambuco. Brasília. Ministério da Saúde; 2016. Disponível em: http://tabnet.datasus.gov.br/cgi/tabcgi. exe?sih/cnv/nrpe.def [2018 julho].

11. Brasil. Ministério da Saúde. Portaria no 2.681, de 7 de novembro de 2013. Redefine o Programa Academia da Saúde no âmbito do Sistema Único de Saúde (SUS) [portaria na internet]. Diário Oficial da União 8 de novembro de 2013 [acesso 2019 abr 02]. Disponível em: http://bvsms.saude.gov. br/bvs/saudelegis/gm/2013/prt2681_07_11_2013.html.

12. Brasil. Ministério da Saúde. Portaria no 2.684, de 8 de novembro de 2013. Redefine as regras e os critérios referentes aos incentivos financeiros de investimento para construção de polos e de custeio no âmbito do Programa Academia da Saúde e os critérios de similaridade entre Programas [portaria na internet]. Diário Oficial da União 9 de novembro de 2013 [acesso 2019 abr 02]. Disponível em: http://bvsms.saude.gov. br/bvs/saudelegis/gm/2013/prt2684_08_11_2013.html.

13. Instituto Brasileiro de Geografia e Estatística [Internet]. Cidades; 2016. [citado em 2018 dez. 02]. Disponível em: http://www.cidades.ibge.gov.br/xtras/perfil. php? codmun $=260190$ \&lang $=$.

14. DATASUS. Cadastro Nacional de Estabelecimentos de Saúde. Consulta Estabelecimento - Identificação. Ministério da Saúde; 2016.Disponível em: <http://cnes.datasus.gov.br/ pages/estabelecimentos/consulta.jsp> [2018 julho].

15. Brasil. Ministério da Saúde. Secretaria de Atenção à Saúde. Portaria $n^{\circ}$ 2.436, de 22 de setembro de 2017. Aprova a Política Nacional de Atenção Básica [portaria na internet]. Diário Oficial da União 23 de setembro de 2017[acesso em 15 de janeiro de 2019]. Disponível em: https://bvsms.saude. gov.br/bvs/saudelegis/gm/2017/prt2436_22_09_2017.html.

16. Brasil. Ministério da Saúde. Portaria n².446 de 11 de novembro de 2014. Política Nacional de Promoção da Saúde - PNPS: revisão da Portaria MS/GM n ${ }^{\circ}$ 687, de 30 de março de 2006. [portaria na internet]. Diário Oficial da União 12 de novembro de 2014 [acesso 2019 jan 15]. Disponível em: http://bvsms.saude.gov.br/bvs/publicacoes/pnps_revisao_ portaria_687.pdf.

17. Silva LMV, Hartz ZMA, Chaves SCM, Silva GAP. Metodologia para análise da implantação de processos relacionados à descentralização da atenção à saúde no Brasil. In: Hartz ZMA, Silva LMV, organizadoras. Avaliação em saúde: dos modelos teóricos à prática na avaliação de programas e sistemas de saúde. $1^{\mathrm{a}}$ ed. Salvador: EDUFBA/ Rio de Janeiro: Editora Fiocruz; 2005. p. 207-53.

18. Jannuzzi PDM. Monitoramento e avaliação de programas sociais: uma introdução aos conceitos e técnicas. $1^{\mathrm{a}}$ ed. Campinas: Editora Alínea, 2016.

19. Sancho LG, Dain S. Avaliação em Saúde e Avaliação Econômica em Saúde: introdução ao debate sobre seus pontos de interseção. Cien Saude Colet. 2012;17(3): 765-74.

20. Guarda FRB, Silva RN, Feitosa WMN, Santos Neto PM, Araújo Júnior J. Caracterização das equipes do Programa Academia da Saúde e do seu processo de trabalho. Rev Bras Ativ Fis Saúde. 2015;20(6):638-40.

21. Brasil EGM, Silva RM, Silva MRF, Rodrigues DP, Queiroz MVO. Promoção da saúde de adolescentes e Programa Saúde na Escola: complexidade na articulação saúde e educação. Rev. esc. enferm. 2017;51(1):3230-76.

22. Guarda FRB, Silva RN, Feitosa WMN, Farias J, Santos Neto PM, Araújo Júnior J. Self-perception of the objective, object and work products of Physical Education Professionals belonging to the Academia da Saúde Programa. Rev Bras Ativ Fis Saude. 2016;21(5):400-9. 
23. Ministério da Saúde, Universidade Federal de Santa Catarina. Curso de aperfeiçoamento: implementação da política de promoção da saúde: programa academia da saúde. Brasília: Ministério da Saúde; 2015. Disponível em:<

Recebido: $12 / 07 / 2020$ http://189.28.128.100/dab/docs/portaldab/documentos/ Aprovado: 07/12/2020 curso_aperfeicoamento_academia.pdf>[2019 janeiro].

\section{Como citar este artigo:}

Silva RN, Oliveira JR, Carneiro RCB, Farias SJM, Guarda FRB. Avaliação do grau de implantação do Programa Academia da Saúde no município de Bezerros, Pernambuco. Rev Bras Ativ Fís Saúde. 2020;25:e0170. DOI: 10.12820/rbafs.25e0170 